\title{
Typical Services and Applications in Smart Cities
}

\author{
Mircea Popa ${ }^{1, *}$, Răzvan Bogdan ${ }^{1}$, Anca Sorana Popa ${ }^{2}$ \\ ${ }^{1}$ Department of Computers and Information Technology, Politehnica University of Timisoara, Timisoara, Romania \\ ${ }^{2}$ Department of Mechatronics, Politehnica University of Timisoara, Timisoara, Romania
}

Copyright $(2016$ by authors, all rights reserved. Authors agree that this article remains permanently open access under the terms of the Creative Commons Attribution License 4.0 International License

\begin{abstract}
One of the big challenges in our days is the rate of the urbanization which leads to an over congestion of cities. It is necessary to develop a new kind of city, based on high-tech technologies. This solution was called Smart City. In this type of city all the necessary tools and methods for continuous monitoring of the state of the city, in all the directions, for an efficient consumption of the resources, for modern health and education, for comfortable living conditions, for fluent transportation, care for the environment and for social and government problems have to be implemented. The present paper gives an overview of them and details a typical service/application consisting in a smart lighting system for a street.
\end{abstract}

Keywords Smart City, Services, Smart Lighting, Energy Consumption

\section{Introduction}

One of the big challenges in our days is the rate of the urbanization which leads to an over congestion of cities. For the first time, in 2007 the number of population living in cities was equal with the number of population living in rural areas. According to [1], $60 \%$ of the population will live in cities until 2030 and according to [2], 80\% of the population from the Western countries will live in cities. Cities are important agglomerations from the economic point of view too. At the level of 2009 , they generated around $80 \%$ of economic production, required $70 \%$ of global energy and 34 cities were part of the World's Top 100 Economies, [2]. Such a population density with such intense economic activity raises some problems: pollution, resource usage, living conditions, transportation etc.

Researches were made in the mentioned directions and the conclusion was that those problems can't be solved individually. It is necessary to develop a new kind of city, based on high-tech technologies. This solution was called Smart City. In this type of city its state is continuously monitored for an efficient consumption of the resources, for modern health and education, for comfortable living conditions, for fluent transportation, caring for the environment and for social and government problems.

One of the layers of a smart city is the layer of Services, which includes typical applications. The present paper gives an overview of them and details a typical service/application consisting in a smart lighting system for a street.

The rest of the paper is organized as follows. The next section describes the main structure of a smart city, the third section presents services/applications, the fourth section describes a smart lighting system for a street and the last section outlines conclusions.

\section{Structure of a Smart City}

There are several ways of dividing smart cities and several ways to structure them.

In [3], smart cities are divided in top-down and bottom-up. In the first category the accent is put on the efficiency in ecology (pollution, energy consumption, emissions, transport) and economy (competitive, support for talented people). The technology is the most important tool. In the second category the accent is put on knowledge and social parameters in order to provide to the citizens a better quality of live and to make them to participate to the knowledge society. The inductive and innovative potential of people to create new solutions is the most important tool.

In [4], it is shown that there are two models for developing smart cities: greenfield and brownfield. The greenfield model corresponds to new cities, built from scratch, created smart from the beginning. This model is applied also in case of new smart neighborhoods or new smart cities in suburban areas corresponding to the idea of "city within a city". The brownfield model consists in making smart an existing city. This can be done in incremental steps, focusing on priorities and drivers.

In [5], the model is made by 5 layers. From bottom to top they are: Natural environment (including topography, flora and fauna and resources), Infrastructure (including land use, roads, buildings and utilities), Resources (including water, oil, air and minerals), Services (including energy, water, transport and services) and Social systems (including people, commerce, culture and policy). 
A city model is presented in [6]. The city was divided into 3 service sectors: Infrastructure services (energy and electricity, water, buildings, transport and infrastructure, waste and data and telecommunications), Community services (healthcare, education, recreation and commerce, travel and transport, security and safety and living environment) and Non-community services (finance, workplaces and persuasive information).

In [7], the Smart City is modelled through 4 layers: Infrastructure (sensors, networks and storage possibilities), E-governance (legislation, rules and policies), Services (energy management, healthcare, water and waste management) and Stakeholders (citizens, government, officials and operators).

In [8] it is shown that a Smart City is made by 4 layers: sensor (collects information and sends it to a central node), network (gives access to networks and information exchange and transfer through public and private networks), platform (ensures the information processing and control) and application.

A different approach is presented in [9]. Unlike the above described solutions which supposed a vertical alignment between the layers, this solutions describes a smart city as a collection of six main characteristics: smart economy, meaning competitiveness, smart people, addressing the social and human capital, smart governance, referring to participation, smart mobility, focusing transport and ICT, smart environment, with accent on natural resources and smart living, pointing out the quality of life. If in the above described solutions the service/applications layer was well delimitated, here services/applications are distributed and embedded in most of the characteristics and their factors.

\section{Typical Services and Applications}

Next, several typical services and applications in smart cities will be described.

In [2], three projects are described: a smart energy project, having the goals to generate green power from solar/wind resources, to fuel-fill electric cars, to supply power for buildings, to reduce pollution and energy costs; an automation project having the goals to use the sunlight to heat up the building and to reduce pollution and energy costs and a health care project having the goals to ensure the movement of the ambulance in the minimum time to an accident and, then, to fast drive to the most appropriate hospital.

In [10] a project in the area of Intelligent Transportation Systems is presented. The fast growth of the number of vehicles combined with the much slower growth of the road infrastructure generates many problems: high pollution, high fuel consumption, high probability of accidents, time waste in intersections which leads to economic loses. There are several solutions: intelligent cars, able to communicate among them, intelligent roads, able to communicate with the intelligent cars, traffic management systems and others. The paper presents a proactive solution for controlling vehicular traffic based on monitoring the traffic flow, analyzing data traffic levels and taking proactive decisions based also on historical traffic data.

A similar application is presented in [11]. The application consists in a map where the current position of the user is indicated. Users can register their destination and can ask for routes or the time of arrival for a specific bus. The traffic was optimized taking into account information from sensors and cameras around the city.

Another application in the same area is the smart parking. The parking slots have sensors being able to communicate with cars and motorists announcing if the parking slot is available. A free parking slot can be detected quickly, saving time and fuel.

A classification of applications, based on IoT, is presented in [12]. According to it, a smart city has services and applications in: eHealth, smart environment, home automation, smart water, smart metering, retail, industrial control, smart animal and farming, smart agriculture, smart logistics, security and emergencies.

In [13] services and applications from existent smart cities are divided in:

- E-government services: public complaints, administrative procedures, job searches, public procurement;

- E-democracy services: dialogue, consultation, polling and voting;

- E-business services: digital marketplaces, tourist guides;

- E-health and telecare services: support to elderly, people with diseases;

- E-security services: public safety, school monitoring, natural hazard management;

- Environmental services: recycling, pollution, noise, waste, energy and water management;

- Intelligent transportation: traffic monitoring, measurement and optimization;

- Communication services: broadband connectivity, digital TV;

- E-learning and e-education services.

Typical applications are presented in [14] where the accent is put on the role of IoT in a smart city. They are part of the application layer and consist in: video monitoring, structural health monitoring, environmental monitoring health monitoring and intelligent transportation.

Similar applications are presented in [15] too. They are divided in: energy management, smart transportation, e-health, video surveillance, waste management, water management, structural health management, smart agriculture, smart homes, security and privacy.

Water management: such a system has the role to avoid water wasting. Due to pipe leakages around $50 \%$ of water is lost. The presence of sensors at different places in pipes has prevented this waste. More than that, intelligent irrigation systems from public parks must be optimized in order to avoid the use of the water when it is not necessary (it rains 
for instance).

Waste management: is an important problem especially in dense populated cities due to the costs and the problem of the storage of garbage. Significant savings, economic and ecological advantages can be obtained putting sensors in the garbage bins. The sensors will detect the level of the load and contribute to the optimization of the collector trucks route. These smart containers have to communicate with a central system which will determine the optimal routes for the collector trucks.

E-health: such a system comprises clinical care, remote monitoring, early intervention, prevention and emergency responses. For that, several solutions were developed. The body area network consist in interconnected sensors placed inside or on a human body, making possible to monitor the physiological parameters remotely, such as electrocardiography, respiration, skin temperature etc. The sensed data can be sent to a medical center to be viewed by a specialist and take decisions in real-time. Another problem optimized with ICT help is the decrease of the emergency response. The surveillance of single elder people is also a major concern. A monitoring system can ask help in case of an in-house accident fastening the emergency intervention.

Video monitoring: consist in surveying people's action in different places (roads, railway stations, bus stations, markets, airports etc.) in order to detect and avoid abnormal and unwanted activity. This requires video cameras, high amount of bandwidth and processing and storage possibilities.

Structural health monitoring: this can be done by embedding sensors in bridges and buildings in order to measure their structural health, the effects of vibrations, the extend of the deformations. This increases the possibility to avoid catastrophic accidents, such as bridge crash, prevents irreversible deterioration of buildings and gives trust to people living in these buildings or visiting it. An economic gain is the prolongation of activities in certain buildings until the moment when the monitoring system allows this. It will be also possible to combine vibration and seismic readings in order to better study and understand the impact of light earthquakes on city buildings.

Smart lighting: this application can be implemented at different levels: at home level, at building for offices, halls and large size institutions level, at street level, at district level and at city level. Such a solution requires the possibility to sense the level of the ambient light and the presence of people and/or cars. It is also necessary to remotely command the on/off state of the light and its level of intensity. A smarter solution anticipates the need of light, taking into consideration the level of the ambient light, the direction of movement of a person or a car and the speed.

Smart homes: a smart home has the role to optimize the utility consumptions while offering very good living conditions. This requires: automatic control of appliances, control of light, control of HVAC, automatic alarm and security system and a customized entertainment system. The energy consumption must be maintained at its lowest value and e-health features must be developed too: regular health check-ups, prediction of the disease, proactive measurements to cure and reporting of any emergency situation. Communication facilities are important: the owner of the smart house has to be able to monitor and control its facilities remotely, being placed anywhere.

Energy management: can be developed at independent house level and at city level. In the first case intelligent outlets will offer information about the energy consumption of different appliances at different time periods. A model can be built for optimizing the in house energy consumption. At the city level, the energy consumption will be measured with smart meters connected in an advanced metering infrastructure. The consumption will be measured remotely and a two-way communication between the supplier and the consumer could be developed. Another important approach is the use of the renewable energy. Solutions were implemented using solar, wind, thermal, biomass and vibration-based energy.

Environmental monitoring: this is absolutely necessary in order to keep under control and reduce one of the more important threats of people that is the pollution. The air quality has to be monitored by sensing parameters such as temperature, humidity, carbon dioxide level etc. especially in crowded areas but also in parks, streets etc. This can lead to establishing a pollution map which will help people to avoid certain areas, at certain moments of the day, in their everyday activities. This will help also people doing sport to choose the green routes. Furthermore another goal can be reached: to study the relationship between human behavior and weather in an attempt to better understand the dynamics of an urban system. Environmental monitoring helps also to control and reduce acoustic pollution. The idea is to reduce the noise level in city areas at specific time periods under a certain level. The environmental monitoring is an important tool for implementing the 20-20-20 Renewable Energy Directive of the European Union. This means a $20 \%$ reduction in greenhouse emissions, a $20 \%$ cut in energy consumption through improved energy efficiency and a $20 \%$ increase of the use of renewable energy by 2020 compared with 1990 levels.

\section{Smart Street Lighting System}

Next, a smart street lighting system based on a wireless network is proposed. The network of lamps is managed according to the level of the ambient light and the presence of people, the aim being to minimize the energy consumption.

Each lamp is controlled by a node composed by a microcontroller, a light sensor, two motion sensors and a wireless networking module. The node will command the lamp according to the level of ambiental light measured. The light can be turned off or on with low, medium, intense levels of intensity. The thresholds are, according to the specialty literature, 1 lux, 50 lux, 1000 lux and 30000 lux. The nodes 
can send wake-up commands to neighbor nodes.

The street model is presented in Fig. 1. The street has a width of $10 \mathrm{~m}$, a length of $225 \mathrm{~m}$ and the distance between the lamps is $25 \mathrm{~m}$. They are mounted at $8 \mathrm{~m}$ above the ground. The maximum beam pattern's length in both directions is 13 $\mathrm{m}$, and its width is $10 \mathrm{~m}$. The pedestrian movement is detected at a maximum distance of $12 \mathrm{~m}$. The lamp is built with the LED technology.

The functionality of the solution was tested with OMNeT++ (Objective Modular Network Testbed in $\mathrm{C}++$ ). The simulation was created for a network of 10 smart nodes with linear topology and a module representing a human moving along the street.

\section{Test case 1}

The simulations were done without pedestrian movement. The simulation conditions were: simulation time of 2 hours, lamps turned on, 2 cases: 1 lux ambiental light, requiring high luminary intensity lighting, and 500 lux ambiental light, requiring medium luminary intensity lighting. The results showed a decrease of $50 \%$ of the energy consumption in the second case (from $55 \mathrm{WH}$ at $28 \mathrm{Wh}$ ).

\section{Test case 2}

The simulations were done with pedestrian movement. The simulation conditions were: simulation time of 2 hours, walking speed $1 \mathrm{~m} / \mathrm{s}$, time for pedestrian passing is between $60 \mathrm{~s}$ and 10 minutes, 2 cases: 1 lux ambiental light and 500 lux ambiental light and lamps turned on at full, respective half capacity.

\section{Test case 2.1}

Only 1 pedestrian is moving along the street from the first position until the last one. We measure the energy consumption at each lamp with 1 lux ambiental light. The results are shown in Table 1.

Table 1. Results for test case 2.1, 1 lux

\begin{tabular}{|c|c|}
\hline Parameter & Value \\
\hline Minimum energy consumption & $0,107 \mathrm{Wh} / \mathrm{LED}$ \\
\hline Maximum energy consumption & $7,84-9,66 \mathrm{Wh} / \mathrm{LED}$ \\
\hline $\begin{array}{c}\text { Comparison with test case 1, 1 lux } \\
\text { ambiental light }\end{array}$ & $80 \%$ reduction \\
\hline $\begin{array}{c}\text { Comparison with test case } 1,500 \text { lux } \\
\text { ambiental light }\end{array}$ & $65 \%$ reduction \\
\hline
\end{tabular}

Table 2 presents the results in the case of 500 lux ambiental light. The minimum values are half of the energy consumption in the previous case and $94 \%$ decrease compared with the case of 500 lux lighting conditions without movement detection.

Table 2. Results for test case 2.2, 500 lux

\begin{tabular}{|c|c|}
\hline Parameter & Value \\
\hline Minimum energy consumption & $0,053 \mathrm{Wh} / \mathrm{LED}$ \\
\hline Maximum energy consumption & $3,92-4,83 \mathrm{Wh} / \mathrm{LED}$ \\
\hline $\begin{array}{c}\text { Comparison with test case } 1,500 \mathrm{lux} \\
\text { ambiental light }\end{array}$ & $94 \%$ reduction \\
\hline
\end{tabular}

Test case 2.2

2 pedestrians are moving along the street. The first human model starts moving from the first led position towards the last one. The second human model moves along the street in the same time but starting at the fourth lamp post. Table 3 shows the results for 1 lux ambiental light.

Table 4 contains the results in case of 500 lux ambiental light. The maximum values are about $50 \%$ of the energy consumption in case of 1 lux and $28 \%$ of the energy consumption in case of 500 lux lighting conditions without moving detection capabilities.

Table 3. Results for test case 2.2, 1 lux

\begin{tabular}{|c|c|}
\hline Parameter & Value \\
\hline Minimum energy consumption & $0,1069-0,1086 \mathrm{Wh} / \mathrm{LED}$ \\
\hline Maximum energy consumption & $7,38-17 \mathrm{Wh} / \mathrm{LED}$ \\
\hline $\begin{array}{c}\text { Comparison with test case } 1,1 \text { lux } \\
\text { ambiental light }\end{array}$ & $69 \%$ reduction \\
\hline $\begin{array}{c}\text { Comparison with test case } 1,500 \text { lux } \\
\text { ambiental light }\end{array}$ & $48 \%$ reduction \\
\hline
\end{tabular}

Table 4. Results for test case 2.2, 500 lux

\begin{tabular}{|c|c|}
\hline Parameter & Value \\
\hline Minimum energy consumption & $0,053 \mathrm{Wh} / \mathrm{LED}$ \\
\hline Maximum energy consumption & $3,69-8,5 \mathrm{Wh} / \mathrm{LED}$ \\
\hline $\begin{array}{c}\text { Comparison with test case } 2.2,1 \\
\text { lux ambiental light }\end{array}$ & $50 \%$ reduction \\
\hline $\begin{array}{c}\text { Comparison with test case } 1,500 \\
\text { lux ambiental light }\end{array}$ & $72 \%$ reduction \\
\hline
\end{tabular}

Test case 2.2 represents an improvement compared with the conditions from Test case 1 but the energy consumption is increased with $40 \%$ compared with Test case 2.1 . 


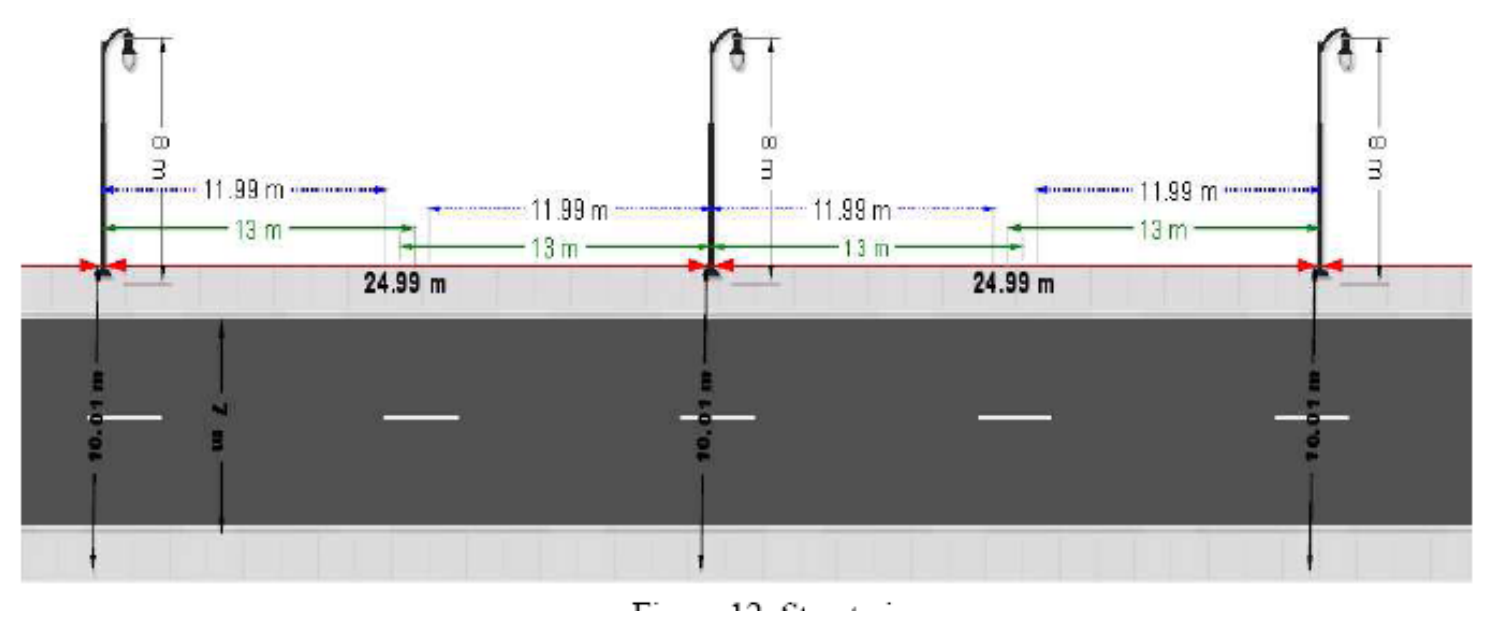

Figure 1. The street model

Test case 2.3

8 pedestrians are moving along the street. The first pedestrian moves from the first led position towards the last one. The following pedestrians move along the street in the same time but starting at consecutive positions.

Table 5. Results for test case 2.3, 1 lux

\begin{tabular}{|c|c|}
\hline Parameter & Value \\
\hline $\begin{array}{c}\text { Minimum energy consumption - } \\
\text { variation }\end{array}$ & $\begin{array}{c}0,85 \mathrm{mWh} / \mathrm{LED} 0-107 \\
\mathrm{mWh} / \mathrm{LED} 9\end{array}$ \\
\hline $\begin{array}{c}\text { Comparison of minimum energy } \\
\text { consumption (average) with test case } \\
2.1 \text { and } 2.2\end{array}$ & $80 \%$ reduction \\
\hline $\begin{array}{c}\text { Maximum energy consumption - } \\
\text { variation }\end{array}$ & $\begin{array}{c}13,3 \mathrm{Wh} / \mathrm{LED} 0-45,3 \\
\mathrm{Wh} / \mathrm{LED} 9\end{array}$ \\
\hline $\begin{array}{c}\text { Comparison of maximum energy } \\
\text { consumption (average) with test case } \\
1,1 \text { lux }\end{array}$ & $40 \%$ reduction \\
\hline $\begin{array}{c}\text { Comparison of maximum energy } \\
\text { consumption (average) with test case } \\
2.1\end{array}$ & 3,5 times greater \\
\hline $\begin{array}{c}\text { Comparison of maximum energy } \\
\text { consumption (average) with test case } \\
2.2\end{array}$ & 2,2 times greater \\
\hline
\end{tabular}

Table 5 illustrates the energy consumption at each LED luminary at 1 lux ambiental light. The minimum values are reduced considerably. The maximum values have also an increasing variation with $40 \%$ lower than the energy consumption in case of 1 lux with 2 hours of artificial constant lighting but 3.5 times greater than 1 lux case from Test case 2.1 and 2.2 times greater than the same case from Test case 2.2.

Table 6. Results for test case 2.3, 500 lux

\begin{tabular}{|c|c|}
\hline Parameter & Value \\
\hline Minimum energy consumption - variation & $\begin{array}{c}0,42 \mathrm{mWh} / \mathrm{LED} 0-53 \\
\mathrm{mWh} / \mathrm{LED} 9\end{array}$ \\
\hline Maximum energy consumption - variation & $\begin{array}{c}6,64 \mathrm{Wh} / \mathrm{LED} 0-22,6 \\
\mathrm{Wh} / \mathrm{LED} 9\end{array}$ \\
\hline Comparison with test case $2.3,1 \mathrm{lux}$ & $50 \%$ reduction \\
\hline Comparison with test case $2.1,500 \mathrm{lux}$ & $70 \%$ more \\
\hline Comparison with test case $2.1,500 \mathrm{lux}$ & $50 \%$ more \\
\hline Comparison with test case $1,500 \mathrm{lux}$ & $40 \%$ reduction \\
\hline
\end{tabular}

Table 6 provides the simulation results in case of 500 lux ambiental light.

For Test case 2.3, the minimum values were much lower than the ones from the other scenarios but the total energy consumption was increased. This is caused by the greater number of pedestrians.

\section{Conclusions}

This paper approached the typical services and applications from a smart city. First the structure of a smart city was presented. Some papers describe smart cities being made by different layers, but others consider it as being defined by main characteristics. In both cases, services and applications have an important role. Then, the main services and applications were described the accent being put on technical aspects. This description helps to create a concrete understanding of what a smart city means and what should be implemented in a city for having smart features. Next, a smart street lighting system based on a wireless network is proposed. The network of lamps is managed according to the level of the ambient light and the presence of people, the aim being to minimize the energy consumption. The simulations were done with $\mathrm{OMNeT++}$. A network of 10 smart nodes with linear topology and a module representing a human moving along the street were considered. The analyzed scenarios were with and without pedestrian movement. In the first case 1, 2 and 8 pedestrian were moving in the same direction. The results showed important decreases in energy consumption by using the smart lighting system: about $50 \%$ without pedestrian movement and more than $48 \%$ with pedestrian movement.

\section{REFERENCES}

[1] M. Naphade, G. Banavar, C. Harrison, J. Paraszczak and R. Morris, Smarter cities and their innovation challenges, in Computer, Volume 44, 2011 
[2] F. A. Reusch, Web of Things-Enabling Smart Cities to reach all potentials, in Proc. Of 25th International World Wide Web Conference, 2016, Montreal, Canada

[3] C. A. Amitrano, A. Alfano and F. Bifulco, New Smart Cities: a focus on some ongoing projects, in Proc. of the 3rd International Virtual Conference, vol. 1, pp. 383-388

[4] J. P. Exner, Smart Planning \& Smart Cities, in Proc. of the 19th International Conference on Urban Planning, Regional Development and Information Society, May 21-23, 2014, Vienna, Austria

[5] C. Harrison and I. A. Donnelly, A Theory of Smart Cities, in Proc. of the $55^{\text {th }}$ Annual Meeting of the ISSS, July 17-22, 2011, Hull, UK

[6] N. Lovehagen, A. Bondesson, Evaluating sustainability of using ICT solutions in smart cities-methodology requirements, in Proc. of the First International Conference on Information and Communication technologies for Sustainability, February 14-16, 2013, Zurich, Switzerland

[7] N. Z. Bawany, J. A. Shamsi, Smart City Architecture: Vision and Challenges, in International Journal of Advanced Computer Science and Applications, Vol. 6, No. 11, 2015

[8] Y. Li, Y. Lin and S. Geertman, The development of smart cities in China, in Proc. of the $14^{\text {th }}$ International Conference on Computers in Urban Planning and Urban Management, July 7-10, 2015, Cambridge MA, USA

[9] R. Giffinger, C. Fertner, H. Kramar, R. Kalasek, N.
Pichler-Milanovic and E. Meijers, Smart cities - Ranking of European medium-sized cities, Centre of Regional Science, October 2007, Vienna, Austria

[10] B. Zavala and G.H. Alferez, Proactive Control of traffic in Smart Cities, in Proc. of the $17^{\text {th }}$ International Conference on Artificial Intelligence, ICAI 15, July 27-30, Las Vegas, USA

[11] O. Hotea and A. Groza, Reasoning on Semantic Sensor Streams for Smart City, in Proc. of the International Conference on Intelligent Information Systems, IIS2013, August 20-23, 2013, Chisinau, Republic of Moldavia

[12] R. Petrolo, V. Loscri and N. Mitton, Towards a Smart City based on Cloud of Things, a survey on the smart city vision and paradigms, in Transactions on Emerging Telecommunication Technologies, March 2015, pp. 1-11

[13] L. Anthopoulos and P. Fitsilis, Using Classification and Roadmapping techniques for Smart City viability's realization, in Electronic Journal of e-Government, Vol. 11, Issue 1, 2013, pp. 326-336

[14] J. Jin, J. Gubbi, S. Marusic and M. Palaniswami, An Information Framework for Creating a Smart City Through Internet of Things, in IEEE Internet of Things Journal, Vol. 1, No. 2, April 2014

[15] S. Mehrotra and R. Dhande, Smart Cities and Smart Homes: From Realization to Reality, in Proc. of the 2015 International Conference on Green Computing and Internet of Things, October 08-10, 2015, Delhi, India 